\title{
Escape routes, weak links, and desynchronization in fluctuation-driven networks
}

\author{
Schäfer, Benjamin; Matthiae, Moritz; Zhang, Xiaozhu; Rohden, Martin; Timme, Marc; Witthaut, Dirk
}

Published in:

Physical Review E

Link to article, DOI:

10.1103/PhysRevE.95.060203

Publication date:

2017

Document Version

Publisher's PDF, also known as Version of record

Link back to DTU Orbit

Citation (APA):

Schäfer, B., Matthiae, M., Zhang, X., Rohden, M., Timme, M., \& Witthaut, D. (2017). Escape routes, weak links, and desynchronization in fluctuation-driven networks. Physical Review E, 95(6), [060203].

https://doi.org/10.1103/PhysRevE.95.060203

\section{General rights}

Copyright and moral rights for the publications made accessible in the public portal are retained by the authors and/or other copyright owners and it is a condition of accessing publications that users recognise and abide by the legal requirements associated with these rights.

- Users may download and print one copy of any publication from the public portal for the purpose of private study or research.

- You may not further distribute the material or use it for any profit-making activity or commercial gain

- You may freely distribute the URL identifying the publication in the public portal 


\title{
Escape routes, weak links, and desynchronization in fluctuation-driven networks
}

\author{
Benjamin Schäfer, ${ }^{1}$ Moritz Matthiae,,${ }^{1,2, *}$ Xiaozhu Zhang, ${ }^{1}$ Martin Rohden, ${ }^{1,3}$ Marc Timme,,${ }^{1,4,5}$ and Dirk Witthaut ${ }^{2,6}$ \\ ${ }^{1}$ Network Dynamics, Max-Planck-Institute for Dynamics and Self-Organization (MPI DS), 37077 Göttingen, Germany \\ ${ }^{2}$ Forschungszentrum Jülich, Institute for Energy and Climate Research-Systems Analysis and Technology Evaluation (IEK-STE), \\ 52428 Jülich, Germany \\ ${ }^{3}$ Jacobs University, Department of Physics and Earth Sciences, 28759 Bremen, Germany \\ ${ }^{4}$ Department of Physics, Technical University of Darmstadt, 64289 Darmstadt, Germany \\ ${ }^{5}$ Institute for Theoretical Physics, Technical University of Dresden, 01062 Dresden, Germany \\ ${ }^{6}$ Institute for Theoretical Physics, University of Cologne, 50937 Köln, Germany
}

(Received 29 November 2016; revised manuscript received 16 March 2017; published 9 June 2017)

\begin{abstract}
Shifting our electricity generation from fossil fuel to renewable energy sources introduces large fluctuations to the power system. Here, we demonstrate how increased fluctuations, reduced damping, and reduced intertia may undermine the dynamical robustness of power grid networks. Focusing on fundamental noise models, we derive analytic insights into which factors limit the dynamic robustness and how fluctuations may induce a system escape from an operating state. Moreover, we identify weak links in the grid that make it particularly vulnerable to fluctuations. These results thereby not only contribute to a theoretical understanding of how fluctuations act on distributed network dynamics, they may also help designing future renewable energy systems to be more robust.
\end{abstract}

DOI: 10.1103/PhysRevE.95.060203

Introduction. The development of a sustainable energy supply is one of the major technical, economical, and societal challenges of the coming decades. To mitigate climate change, the majority of existing fossil-fuel power plants will be replaced by renewable energy sources, in particular wind and solar power [1,2]. This requires a comprehensive reengineering of the electric power grid as well as a better understanding of the operation, dynamics, and stability of complex networked systems [3-6].

The operation of wind turbines and photovoltaics is essentially determined by the weather such that the power generation fluctuates strongly on all time scales [7]. Large storage and backup infrastructures are needed to balance power generation and demand on time scales of hours to months [8]. In addition, the high-frequency fluctuations can be enormous, in particular due to the turbulent character of wind power [9,10]. At the same time, large fossil-fuel plants are closed down such that the effective inertia and damping decreases, making the power grid more vulnerable to instabilities by transients [11].

Here we analyze how high-frequency fluctuations threaten the dynamical robustness of electric power grids. The stable operation of a grid requires all generators and loads to remain phase locked. We analyze the robustness of this phase-locked state, mapping the grid dynamics to an escape problem in a high-dimensional stochastic dynamical system. This analysis reveals essential factors which limit the operability of future power grids. Furthermore, we uncover how the network topology determines the robustness of the grid and identify potential escape routes and vulnerable links in complex networks.

Synchronization and robustness of electric power grids. Consider first the dynamics of coupled synchronous genera-

*Current address: Department of Micro- and Nanotechnology, Technical University of Denmark, 2800 Kongens Lyngby, Denmark. tors and consumers $j \in\{1, \ldots, N\}$. Each unit's dynamics is described by the swing equation [12-14] for the mechanical rotor angle $\delta_{j}$ and the phase velocity $\omega_{j}$ relative to the grid reference frequency $\Omega=2 \pi \times 50$ (or 60 ) $\mathrm{Hz}$,

$$
\begin{gathered}
\dot{\delta}_{j}=\omega_{j}, \\
\frac{2 H_{j}}{\Omega} \dot{\omega}_{j}+2 \Omega D_{j} \omega_{j}=P_{j}-P_{j}^{(\mathrm{el})},
\end{gathered}
$$

where the right-hand side is the net power acting on the machine. The swing equation is made dimensionless by dividing all terms by the rated power of the machine, which is referred to as the "per unit system" in engineering [12]. The inertia constant $H_{j}$ then gives the stored kinetic energy at synchronous speed which is proportional to the moment of inertia of the $j$ th machine and $D_{j}$ is a damping constant. If not mentioned otherwise, we assume a typical value of inertia $H_{j}=H=4 \mathrm{~s}$ and a damping constant of $D_{j}=D=$ $4 \times 10^{-5} \mathrm{~s}^{2}[15,16]$.

The input power $P_{j}$ driving a machine can be subject to strong fluctuations on various time scales induced by renewable resources [9] or consumer behavior [17]. We thus analyze the robustness of the phase-locked state to stochastic fluctuations $\xi_{j}(t)$, i.e., we set

$$
P_{j}(t)=\bar{P}_{j}+\xi_{j}(t)
$$

The electric power $P_{j}^{(\mathrm{el})}$ exchanged over a transmission line is determined by the difference of the voltage phase angle of the two terminal nodes. For a common two-pole machine the voltage phase angle equals the mechanical phase angle such that the transmitted real power reads $K_{i j} \sin \left(\delta_{i}-\right.$ $\left.\delta_{j}\right)$. The capacity parameter $K_{i j}$, describing the maximally transmittable power on the transmission line between nodes $i$ and $j$, is determined by the susceptance $B_{i j}$ of the line and the voltage levels $E_{i}$ and $E_{j}$ at the two units as $K_{i j}=B_{i j} E_{i} E_{j}$. We note that $K$ gives the physical limit of the transmitted real 

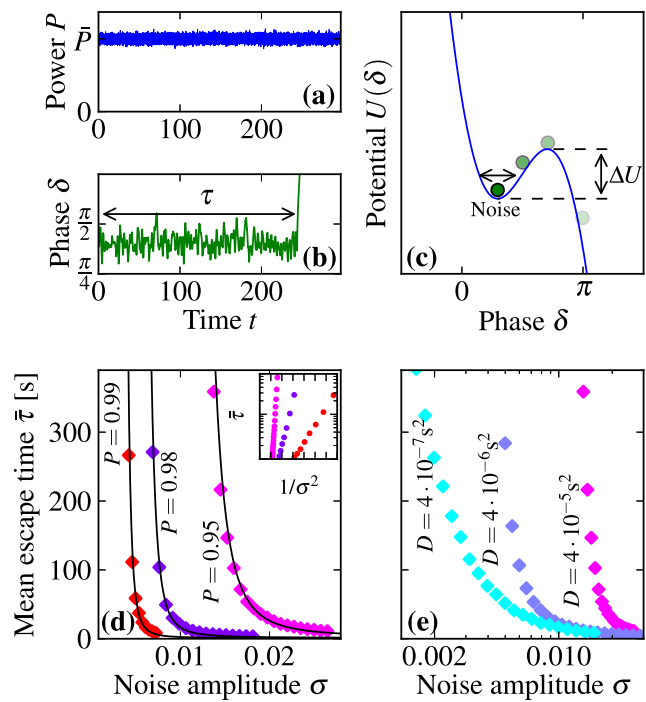

FIG. 1. Fluctuating power input may desynchronize a synchronous generator. (a),(b) When the input power $P$ fluctuates, the generator can lose synchrony to the grid after an escape time $\tau$. (c) The generator dynamics corresponds to the motion of a particle in a tilted washboard. The fluctuations can drive the particle over the potential barrier of height $\Delta U$. (d) Kramer's escape rate theory predicts the escape process for Gaussian white noise. Theoretical prediction, Eq. (5), (black lines) perfectly predicts the mean escape times $\bar{\tau}$ for intermediate damping $\left(D=4 \times 10^{-5} \mathrm{~s}^{2}\right)$ as checked by direct numerical simulations (symbols). The averaged escape time crucially decreases with increasing load $P$ of the system. (e) Weaker damping $D$ undermines system robustness, which can become a major problem in future power grids. (Parameters: $H=4 \mathrm{~s}, K=1$, and $\bar{P}=0.95$, if not stated otherwise.)

power in the lossless case of our dynamical model. Ohmic losses can lead to an overheating of lines on longer time scales leading to the definition of thermal capacity, which includes a security limit by the grid operators $[12,18]$.

In a complex network of lines and generators the total electric power transmitted from machine $j$ is thus given by $P_{j}^{(\mathrm{el})}=\sum_{i} K_{i j} \sin \left(\delta_{i}-\delta_{j}\right)$. Stable operation of the grid requires all units to be in a phase-locked state where $\delta_{i}-\delta_{j}$ is fixed. Otherwise, the transmitted electric power $P_{j}^{(\mathrm{el})}(t)$ would oscillate and average out over time [14,15,19-21]. Throughout this Rapid Communication we assume a heavily loaded grid, where phase differences are comparably large in the stable phase-locked state. Such a situation is yet uncommon, but will become increasingly likely in the future [22]. Other scenarios are analyzed in the Supplemental Material [23], including less heavily loaded transmission lines, inverters without inertia $H$ [24], and higher-order power grid models including voltage dynamics [12,25].

Robustness of a single generator. First, consider a single generator coupled to a large bulk grid. The steady-state operation of the generator is described by a stable phase-locked state, a fixed point of the swing equation with a constant phase difference $\delta$ relative to the grid. Fluctuations of the input power $P$ can destabilize the grid as illustrated in Figs. 1(a) and 1(b). As soon as the generator leaves the basin of attraction of the stable phase-locked state after some time $\tau$ it rapidly desynchronizes. Such a serious contingency requires immediate emergency measures to avoid a global network failure.

We analyze desynchronization due to white noise by Kramer's escape rate theory [26-29] as follows: The equation of motion for the generator is equivalent to a driven dissipative particle moving in a tilted washboard potential [30], i.e., $\ddot{\delta}+\left(\Omega^{2} D / H\right) \dot{\delta}=(\Omega / 2 H)[-d U / d \delta+\xi(t)]$ with the effective potential [31]

$$
U(\delta)=-\bar{P} \delta-K \cos (\delta) .
$$

Thus, to escape the basin of attraction around a local minimum of $U(\delta)$ the particle must overcome a potential barrier of height $\Delta U$ [see Fig. 1(c)], which is determined by the transmitted power $P$ and the capacity $K$. For Gaussian white noise $\xi(t)$ with standard deviation $\sigma$ the mean escape time is given by [27]

$$
\bar{\tau}=\frac{2 \pi \lambda}{\sqrt{U^{\prime \prime}\left(\delta_{\min }\right)\left|U^{\prime \prime}\left(\delta_{\max }\right)\right|}} \exp \left(\frac{2 \gamma \Delta U}{\sigma^{2}}\right),
$$

with effective damping $\gamma=2 D \Omega$ and $2 \lambda=\gamma+$ $\sqrt{\gamma^{2}+(8 H / \Omega)\left|U^{\prime \prime}\left(\delta_{\max }\right)\right|}$ for intermediate damping and $U^{\prime \prime}\left(\delta_{\min }\right)$ and $U^{\prime \prime}\left(\delta_{\max }\right)$ being the second derivatives of the potential evaluated at the local minimum and the saddle point of the potential $U(\delta)$, respectively [27]. Numerical simulations averaged over 2000 escape processes for each value of $\sigma$ show excellent agreement with this prediction [see Fig. 1(d)].

Major concerns about the stability of future power grids arise from the increased transmission needs and lines loads [22] as well as a possible lack of effective inertia and damping when conventional generators are replaced by renewables [11]. Heavily loaded lines are indeed very susceptible to desynchronization as shown in Fig. 1(d). Increasing the load $P$ rapidly decreases the escape time $\bar{\tau}$. Similarly, a decrease of the effective damping factor $D$ implies a rapid decrease of $\bar{\tau}$ [Fig. 1(e)]. In contrast, the inertia $H$ has a minor effect only, as it enters the escape rate (5) only through the nonexponential prefactors [23].

We note that this stability assessment defined by Kramer's escape rate is complementary to existing studies investigating large-scale perturbation in power grids in terms of Lyaponiv exponents [32], basin stability [33], or cascading failures [34-36]. Our stochastic approach focuses on small perturbations eventually driving the grid out of synchrony in contrast to singular large disturbing events.

Escape dynamics in phase space. The essential factors in Kramer's formula (5) are the amplitude of the noise $\sigma$, the effective damping $\gamma$, and the height of the potential barrier $\Delta U$. The theory of random dynamical systems [29] implies that in the limit of weak noise $(\sigma \rightarrow 0)$ the system escapes the basin of attraction in the vicinity of a saddle point, where the potential gap to the stable fixed point is smallest. An exemplary escape process in phase space is shown in Fig. 2 for the single generator system. Intriguingly, we observe that at any nonzero noise level $\sigma>0$, the trajectory leaves the basin near but not exactly at the saddle point (red dot). Only in the limit of small perturbations, i.e., $\sigma \rightarrow 0$, does the system leave the fixed point exactly at the saddle.

The saddle point itself is characterized by a vanishing velocity $d \delta / d t=0$. However, all simulated trajectories leave the basin with a nonvanishing velocity $d \delta / d t>0$ (i.e., 

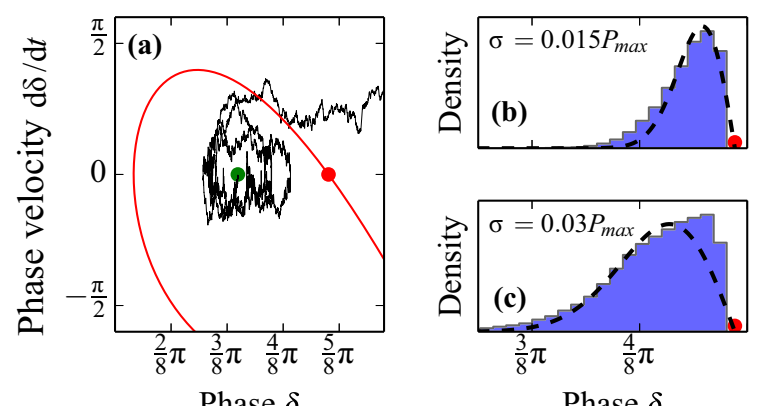

Phase $\delta$

FIG. 2. Where does the system escape? (a) Exemplary escaping process of the basin of attraction in phase space. The state trajectory (thin black line) crosses the boundary of the basin of attraction (red line) in the vicinity of but not at the saddle point (red disk). The stable fixed point is indicated by a green disk. (b),(c) Probability distribution of exit points, i.e., the crossing points of the trajectory and the basin boundary. Numerical results (histogram) compared to the theoretical prediction, Eq. (6) (dashed black line). With increasing noise the distribution becomes broader, i.e., a crossing at some distance from the saddle point (red disk) becomes more probable. (Parameters: $K=1$ and $\bar{P}=0.95$ as in Fig. 1.)

"above" the saddle point in the phase space portrait shown in Fig. 2). More precisely, the probability density of the trajectory on the basin boundary in phase space is given by a Weibull function [37]

$$
p(\delta)=\mathcal{N} \delta \exp \left(-\frac{\lambda^{2} \delta^{2}}{2 \sigma^{2}}-\frac{2 \Omega}{H} \frac{\left|U^{\prime \prime}\left(\delta_{\max }\right)\right| \delta^{2}}{2 \sigma^{2}}\right),
$$

where $\mathcal{N}$ is a normalization constant. This theoretical prediction is equally well confirmed by the numerical simulations as shown in Fig. 2 on the right. With increasing noise amplitude the distribution gets broader, i.e., the escape velocity increases.

Escape via the weakest link. To maintain a stable operation it is essential to know not only under which conditions, but also how power system operation may become unstable. We first consider a simple system of two identical generators coupled to a bulk power grid with transmission lines of different capacity, both being subject to independent and identically distributed Gaussian white noise [see Fig. 3(a)]. Either of the two generators can become unstable, such that the grid can escape the basin of attraction of the stable phase-locked state via two different routes. Scaling of the mean escape time is still described by Kramer's rate for intermediate damping, when we take into account that the lower potential barrier along both routes determines the escape [see Fig. 3(b)]. The two-dimensional potential is then given as

$$
U\left(\delta_{1}, \delta_{2}\right)=-\bar{P}_{1} \delta_{1}-K_{1} \cos \left(\delta_{1}\right)-\bar{P}_{2} \delta_{2}-K_{2} \cos \left(\delta_{2}\right)
$$

In the limit of weak noise the escape problem is fully determined by the path with the smallest potential barrier $\Delta U$. To illustrate this we vary the capacity $K_{2}$ of the transmission line connecting generator 2 to the bulk grid while the capacity $K_{1}$ of the other line remains fixed. For $K_{2}<K_{1}$ the robustness is dominated by generator 2 , whose connection is weaker. The exponent in Kramer's formula (5) then crucially depends on the value of $K_{2}$. Indeed, the exponent obtained from the numerical
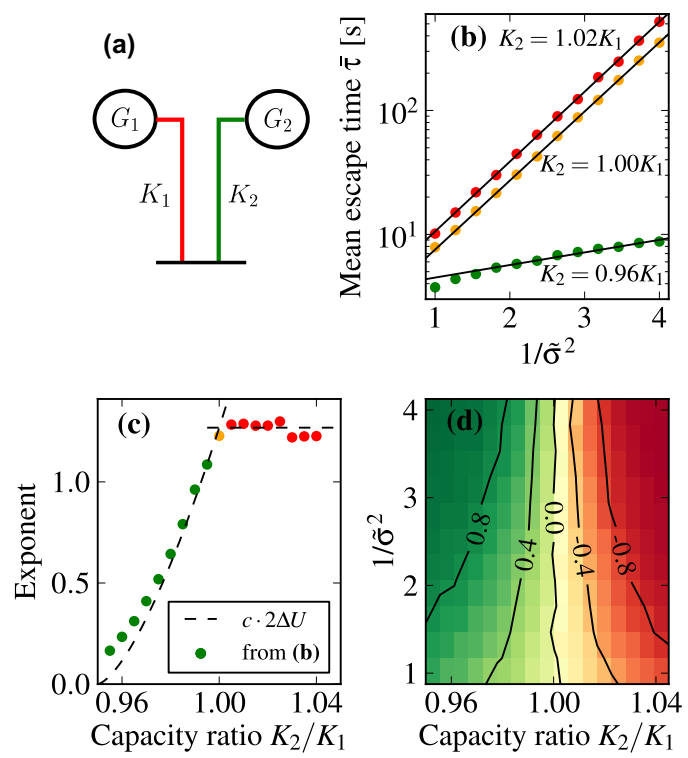

FIG. 3. The easiest escape route determines the escape time $\bar{\tau}$. (a) Two identical generators are coupled to a third node representing the bulk grid via transmission lines with capacity $K_{1}=1$ (constant) and $K_{2}$ (variable), respectively. The transmitted power on both lines is $\bar{P}_{1,2}=0.95$. Power fluctuates on all nodes independently. (b) The mean escape time $\bar{\tau}$ as a function of the noise amplitude $\sigma$. Disks represent numerical values; the solid lines are fits to extract the scaling exponent. (c) In this scenario, the exponent in Kramer's rate is determined by the lowest barrier $\Delta U$, Eq. (7) of the two-dimensional potential landscape, which is determined by $\min \left\{K_{1}, K_{2}\right\}$, i.e., the weaker of the two transmission line capacities. Thus it increases with $K_{2}$ as long as $K_{2} \leqslant K_{1}$ but depends only on $K_{1}$ for $K_{2}>K_{1}$. A comparison of numerical results obtained from exponential fits to the data (disks) and the analytical value of the potential barrier $\Delta U$ (with constant $c$ ) shows very good agreement. (d) Imbalance of the two escape routes: $p_{2}-p_{1}$ with $p_{1,2}$ being the probability that link 1 or 2 is overloaded first, as a function of $K_{2} / K_{1}$ and the noise amplitude. For weak noise there is a sharp transition at $K_{2} / K_{1}=1$, which smears out for stronger noise. Panels (b)-(d) use a rescaled noise $\tilde{\sigma}=40 \sigma$.

simulations again matches the theoretical predictions well in terms of the potential barrier $\Delta U$ [see Fig. 3(c)]. If we increase $K_{2}$ beyond $K_{1}$, the other transmission line becomes the Achilles' heal of the grid. The potential barrier and hence the exponent in Kramer's formula thus no longer depend on $K_{2}$. Yet, the nonexponential prefactor in the formula (5) increases by increasing $K_{2}$ further because the relative transmission line load of the overall system decreases. When the noise becomes stronger, the sharp transition between the two possible escape routes gradually blurs, such that the more strongly connected generator can become unstable too [see Fig. 3(d)].

Robustness of complex power grids. In power grids with a less simple structure, it is essential to understand how the topology determines robustness and to identify possible routes of instability. This enables a precise improvement of the grid and the elimination of weak links. Figure 4(a) shows the stable fixed point in a grid with four generator and eight consumer nodes. The consumer dynamics also follows Eq. (2), but with $P_{j}<0$. A fluctuating input can lead to a loss of synchrony and eventually to a system-wide failure. But where does this 


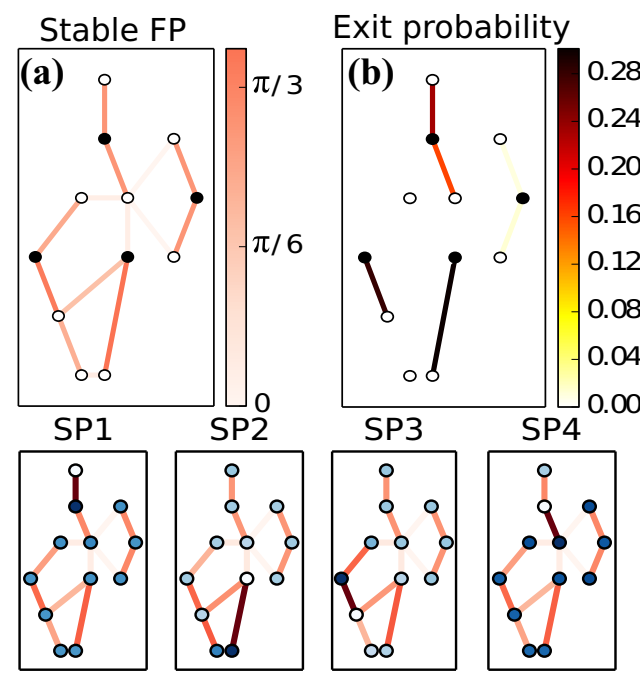

FIG. 4. Vulnerable links predicted by the topology of saddle points. Top: (a) The stable phase-locked fixed points in a model power grid with four generators (filled circles) and eight consumers (open circles). Shown is the phase difference $\delta_{i}-\delta_{j}$ along the transmission lines. (b) Probability that a transmission line is overloaded first $\left(\left|\delta_{i}(t)-\delta_{j}(t)\right|\right.$ crosses $\left.\pi / 2\right)$ when the grid becomes unstable due to a fluctuating power input. Four vulnerable transmission lines are identified. Bottom: The vulnerable transmission lines can be traced back to four different saddle points with comparably low potential barrier. All saddle points have exactly one transmission line (darkest line in each plot) with $\left|\delta_{i}-\delta_{j}\right|>\pi / 2$, corresponding to the vulnerable lines identified in (b). The color scale shows the phase differences as in panel (a). The networks consist of four generators $\left(\bullet, P_{j}=+2 P_{0}\right)$ and eight consumers $\left(\circ, P_{j}=-P_{0}\right)$; all lines have capacity $K=24 / 19 \times P_{0}$.

instability emerge and which of the transmission lines is most vulnerable?

We simulate the dynamics with all machines subject to independent and identically distributed white noise and record which transmission line becomes overloaded first, i.e., we record for which link $(i, j)$ the phase difference $\left|\delta_{i}-\delta_{j}\right|$ first crosses $\pi / 2$. In this way we identify four transmission lines which are vulnerable. Strikingly, these vulnerable lines are not necessarily the ones which are most heavily loaded in the first place. The loss of synchrony in a complex grid is a collective process, which cannot fully be understood from fundamental properties of single nodes or lines [38,39].

Instead, Kramer's theory tells that the saddle points of the entire dynamical system are decisive: As above, the grid leaves the basin of attraction of the stable phase-locked state in the vicinity of the saddle points. In a complex network, many saddle points may exist. But for the application of Kramer's theory we only need to consider those saddle points with the lowest potential barrier, as escape through all other saddle points is exponentially suppressed. For the system studied here, these saddle points are calculated systematically using a method introduced in [40]. This method classifies the saddle points by the number of links $(i, j)$ where the phase difference $\left|\delta_{i}-\delta_{j}\right|$ exceeds $\pi / 2$. Typically, the higher this number, the higher is the potential barrier.

For the sample network depicted in Fig. 4 for illustration, this method yields four saddle points with a comparably low potential barrier, all contributing to the escape process (four lower panels). All four saddles have exactly one line where the phase difference $\left|\delta_{i}-\delta_{j}\right|$ exceeds $\pi / 2$. The static analysis thus yields four vulnerable lines which exactly match the lines where overloads have been recorded in the numerical simulations. Even more, Kramer's rate with the respective barrier heights again predicts the exit probabilities (not shown).

Conclusion. In this Rapid Communication we have analyzed how high-frequency fluctuations impact the dynamical robustness of electric power grids. Focusing on Gaussian white noise yielded analytical access, thereby providing deeper insights into the collective dynamics of fluctuation-driven networks. To characterize the robustness of this stochastic system, we derived the scaling of escape times as a function of the grid load, inertia, damping, and the noise amplitude. These analytic results are applicable in the dimensioning of future renewable power grids, where effective inertia and damping must be provided by power electronic devices. Remarkably, the inertia $H$ enters the escape time only algebraically, whereas the damping enters exponentially. While the assumption of Gaussian white noise is common when investigating power grids under uncertainty, we go beyond the typical restriction to case studies [41] by providing analytical insight. Furthermore, we demonstrated how power networks may escape the regime of stable operation. The grid escapes in the vicinity of (saddle) fixed points with a low potential barrier. Interestingly, these can typically be assigned to a single overloaded link, thus revealing the weak links of the grid.

Complementary work on power grid fluctuations [25] addresses the impact of intermittent noise and incorporates features of real wind turbines. Such settings avert the analytic treatment in terms of Kramer's escape theory. The analytic approach presented in this Rapid Communication reveals which factors limit the robustness of power grid operation to fluctuating inputs. The results may thus not only provide efficient methodology to analyze fluctuation-driven oscillatory systems but may also help planning grid extensions to assure dynamic stability and robustness in future highly renewable power systems.

Acknowledgments. We gratefully acknowledge support from the Federal Ministry of Education and Research (BMBF Grant No. 03SF0472A-E), the Helmholtz Association (via the joint initiative "Energy System 2050-A Contribution of the Research Field Energy" and Grant No. VH-NG-1025 to D.W.), the Göttingen Graduate School for Neurosciences and Molecular Biosciences (DFG Grant No. GSC 226/2 to B.S.), and the Max Planck Society (M.T.).
[1] S. M. Amin and B. F. Wollenberg, IEEE Power Energy Mag. 3, 34 (2005).
[2] T. Bruckner et al., in Climate Change 2014: Mitigation of Climate Change. Contribution of Working Group III to the Fifth 
Assessment Report of the Intergovernmental Panel on Climate Change, edited by O. Edenhofer et al. (Cambridge University Press, Cambridge, UK, 2014).

[3] S. H. Strogatz, Nature (London) 410, 268 (2001).

[4] E. Marris, Nature (London) 454, 570 (2008).

[5] C. D. Brummitt, P. D. H. Hines, I. Dobson, C. Moore, and R. M. D’Souza, Proc. Natl. Acad. Sci. USA 110, 12159 (2013).

[6] M. Timme, L. Kocarev, and D. Witthaut, New J. Phys. 17, 110201 (2015).

[7] R. Sims et al., in IPCC Special Report on Renewable Energy Sources and Climate Change Mitigation, edited by O. Edenhofer et al. (Cambridge University Press, Cambridge, UK, 2011).

[8] D. Heide, L. Von Bremen, M. Greiner, C. Hoffmann, M. Speckmann, and S. Bofinger, Renewable Energy 35, 2483 (2010).

[9] P. Milan, M. Wächter, and J. Peinke, Phys. Rev. Lett. 110, 138701 (2013).

[10] M. Anvari, G. Lohmann, M. Wächter, P. Milan, E. Lorenz, D. Heinemann, M. R. R. Tabar, and J. Peinke, New J. Phys. 18, 063027 (2016).

[11] A. Ulbig, T. S. Borsche, and G. Andersson, IFAC Proc. Vol. 47, 7290 (2014).

[12] J. Machowski, J. Bialek, and J. Bumby, Power System Dynamics, Stability and Control (Wiley, New York, 2008).

[13] G. Filatrella, A. H. Nielsen, and N. F. Pedersen, Eur. Phys. J. B 61, 485 (2008).

[14] M. Rohden, A. Sorge, M. Timme, and D. Witthaut, Phys. Rev. Lett. 109, 064101 (2012).

[15] A. E. Motter, S. A. Myers, M. Anghel, and T. Nishikawa, Nat. Phys. 9, 191 (2013).

[16] T. Nishikawa and A. E. Motter, New J. Phys. 17, 015012 (2015).

[17] A. J. Wood, B. F. Wollenberg, and G. B. Sheblé, Power Generation, Operation and Control (Wiley, New York, 2013).

[18] P. Kundur, N. J. Balu, and M. G. Lauby, Power System Stability and Control (McGraw-Hill, New York, 1994), Vol. 7.

[19] M. Rohden, A. Sorge, D. Witthaut, and M. Timme, Chaos 24, 013123 (2014).

[20] F. Dörfler, M. Chertkov, and F. Bullo, Proc. Natl. Acad. Sci. USA 110, 2005 (2013).
[21] B. Schäfer, M. Matthiae, M. Timme, and D. Witthaut, New J. Phys. 17, 015002 (2015).

[22] T. Pesch, H.-J. Allelein, and J.-F. Hake, Eur. Phys. J.: Spec. Top. 223, 2561 (2014).

[23] See Supplemental Material at http://link.aps.org/supplemental/ 10.1103/PhysRevE.95.060203 for discussion of robustness of the phenomena reported and a comparison of the escape time across different power grid models.

[24] J. W. Simpson-Porco, F. Dörfler, and F. Bullo, IFAC Proc. Vol. 45, 264 (2012).

[25] K. Schmietendorf, O. Kamps, and J. Peinke, arXiv:1611.08235.

[26] H. A. Kramers, Physica 7, 284 (1940).

[27] N. van Kampen, Stochastic Processes in Physics and Chemistry (Elsevier, Amsterdam, 1992).

[28] P. Hänggi, P. Talkner, and M. Borkovec, Rev. Mod. Phys. 62, 251 (1990).

[29] C. W. Gardiner, Handbook of Stochastic Methods for Physics, Chemistry, and the Natural Sciences (Springer, Berlin, 2004).

[30] H. Risken, The Fokker-Planck Equation (Springer, Berlin, 1984).

[31] D. Manik, D. Witthaut, B. Schäfer, M. Matthiae, A. Sorge, M. Rohden, E. Katifori, and M. Timme, Eur. Phys. J.: Spec. Top. 223, 2527 (2014).

[32] P. Kundur, J. Paserba, V. Ajjarapu, G. Andersson, A. Bose, C. Canizares, N. Hatziargyriou, D. Hill, A. Stankovic, C. Taylor et al., IEEE Trans. Power Syst. 19, 1387 (2004).

[33] P. J. Menck, J. Heitzig, J. Kurths, and H. J. Schellnhuber, Nat. Commun. 5, 3969 (2014).

[34] F. Hellmann, P. Schultz, C. Grabow, J. Heitzig, and J. Kurths, Sci. Rep. 6, 29654 (2016).

[35] J.-W. Wang and L.-L. Rong, Safety Sci. 49, 807 (2011).

[36] D. Witthaut and M. Timme, Phys. Rev. E 92, 032809 (2015).

[37] Z. Schuss, Theory and Applications of Stochastic Processes-An Analytical Approach (Springer, New York, 2010).

[38] D. Witthaut, M. Rohden, X. Zhang, S. Hallerberg, and M. Timme, Phys. Rev. Lett. 116, 138701 (2016).

[39] P. Crucitti, V. Latora, and M. Marchiori, Physica A (Amsterdam) 338, 92 (2004).

[40] D. Manik, M. Timme, and D. Witthaut, arXiv:1611.09825.

[41] X. Fang, S. Misra, G. Xue, and D. Yang, IEEE Commun. Surveys Tutorials 14, 944 (2012). 